\title{
Super-Resolution Imaging of Drug Delivery to Live Cells using Real-Time Structured Illumination Microscopy
}

\author{
J. Lefman, ${ }^{* * *}$ T. Cheuk, ${ }^{*}$ B. Metaferia, ${ }^{*}$ K. Scott, ${ }^{* *}$ J. Khan, ${ }^{*}$ and S.J. Stranick**
}

* Oncogenomics Section, Pediatric Oncology Branch, Center for Cancer Research, National Cancer Institute, National Institutes of Health, Gaithersburg, MD, 20877, USA.

** Surface and Microanalysis Division, National Institute of Standards and Technology, Gaithersburg, MD, 20899, USA.

Imaging the effects of drug delivery in live cells is most often performed by confocal microscopy, which has a resolution limited by the diffraction of light [1]. Here, we report the development of a novel method for imaging drug delivery to live cells using real-time super-resolution microscopy in wide-field mode. Structured illumination microscopy (SIM) is one of several super-resolving microscopy techniques and is capable of imaging beyond the resolution of conventional microscopy to less than $100 \mathrm{~nm}[2]$.

In SIM, each super-resolution (SR) image is computationally constructed from a dataset of numerous raw images. Due to the computational time required for SR image construction, datasets are usually post-processed to avoid slowdowns during the acquisition process. Although post-processing is convenient, this approach has limited use since SR images are not available during image acquisition and thus cannot be utilized for real-time evaluation of the sample. Utilizing SR images in real-time is particularly important when objects of interest are smaller than the resolution limit of conventional microscopy. When imaging live cells in real-time, image acquisition, processing, and the display of SR images must occur in rapid succession thereby reducing the effects of molecular movement. To achieve the data processing speed necessary for real-time imaging of live cells, we utilized a graphics processing unit (GPU) to perform high-speed SR image construction. Each function for processing SIM data was optimized for parallel operation using GPU kernels. Compared with processing on a multi-core computer (CPU), using the GPU achieved a 45-fold increase in processing speed. Table 1 compares the running times for each operation in SIM processing.

We applied this real-time SIM platform to record the action of $1 \mathrm{mM}$ nocodazole on microtubule depolymerization in HeLa cells. HeLa cells readily grow on glass coverslips prepared with poly-L lysine. Upon delivery of the drug into the flow chamber designed for imaging live cells, microtubule depolymerization is observed within minutes and cells move away from the sectioning plane created by structured illumination (figure 1). These effects are not apparent using conventional resolution imaging (figure 2). The optimal focus value can be reset using a real time SR display and computer controlled piezo-electric focusing.

References:

[1] Gao, X., et al., Curr. Opin. Biotechnol., 2005. 16.

[2] Gustafsson, M.G.L., PNAS, 2005. 102.

[3] Funding for J.L. by National Research Council Research Associateship Programs fellowship. 
Table 1. Running times of processing steps for structured illumination microscopy

\begin{tabular}{llcc} 
Sub-process & Data form (32 bit) & CPU (ms) & GPU (ms) \\
\hline FFT & $512 \times 512$ pixels; 9 images & 80 & 2.8 \\
IFFT & 1024 x 1024 pixels; 9 images & 910 & 10.3 \\
OTF $^{-1}$ & 1024 x 1024 pixels; 9 images & 100 & 2.9 \\
Unwrap high $f$ & 1024 x 1024 pixels; 9 images & 370 & 2.3 \\
Reduce data & 1024 x 1024 pixels; 9 images & 340 & 0.5 \\
Overhead / latency & & - & $\sim 36$ \\
\hline Total & & 2005 & 55
\end{tabular}

Figure 1 . Three images were acquired 0,9 , and 14 minutes after the addition of $1 \mathrm{mM}$ nocodazole to
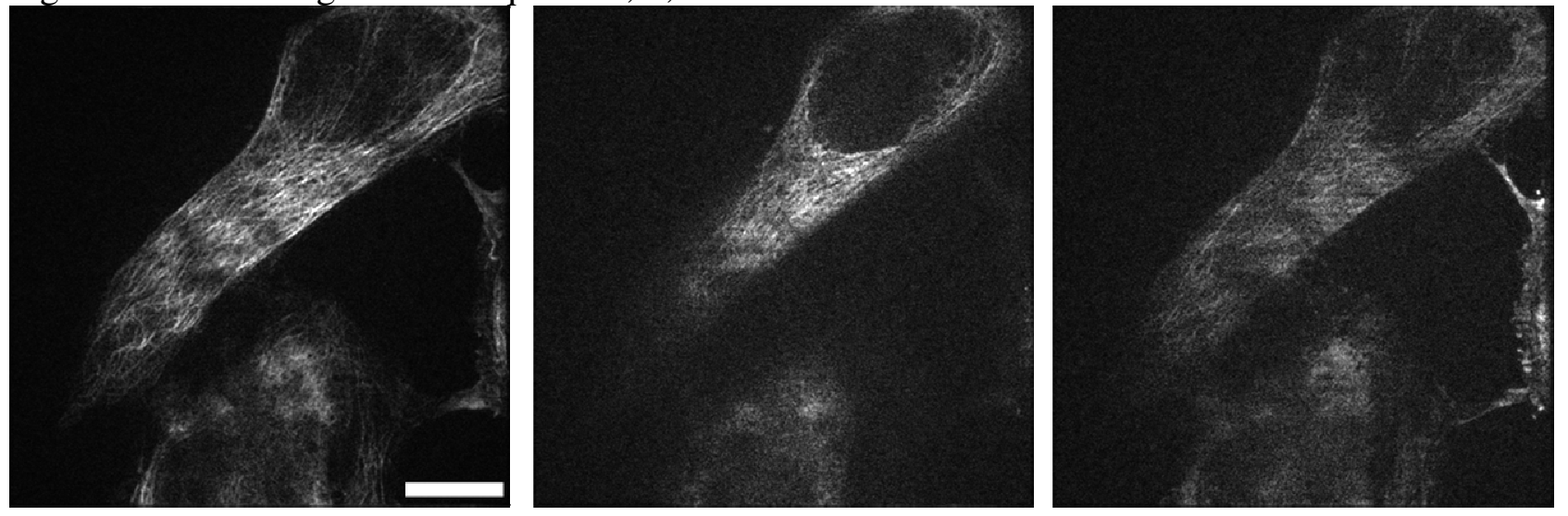

the microscopy flow chamber. There are many microtubule filaments present before the cell retracts as depolymerization of $\alpha$-tubulin occurs. The third image shows restoration of the specimen to the focal plane. The scalebar corresponds to $10 \mu \mathrm{m}$.

Figure 2. To determine the optimum focus value, it is necessary to utilize superresolution data during data acquisition. Here, two super-resolution images, top-left and bottom-left, are compared with corresponding conventional resolution (CR) images on the right. Each SR image displays the region that is in focus. However, the CR images are visually similar and therefore it would be difficult to ascertain the proper focus without real-time SR images. The scalebar corresponds to 10 $\mu \mathrm{m}$.
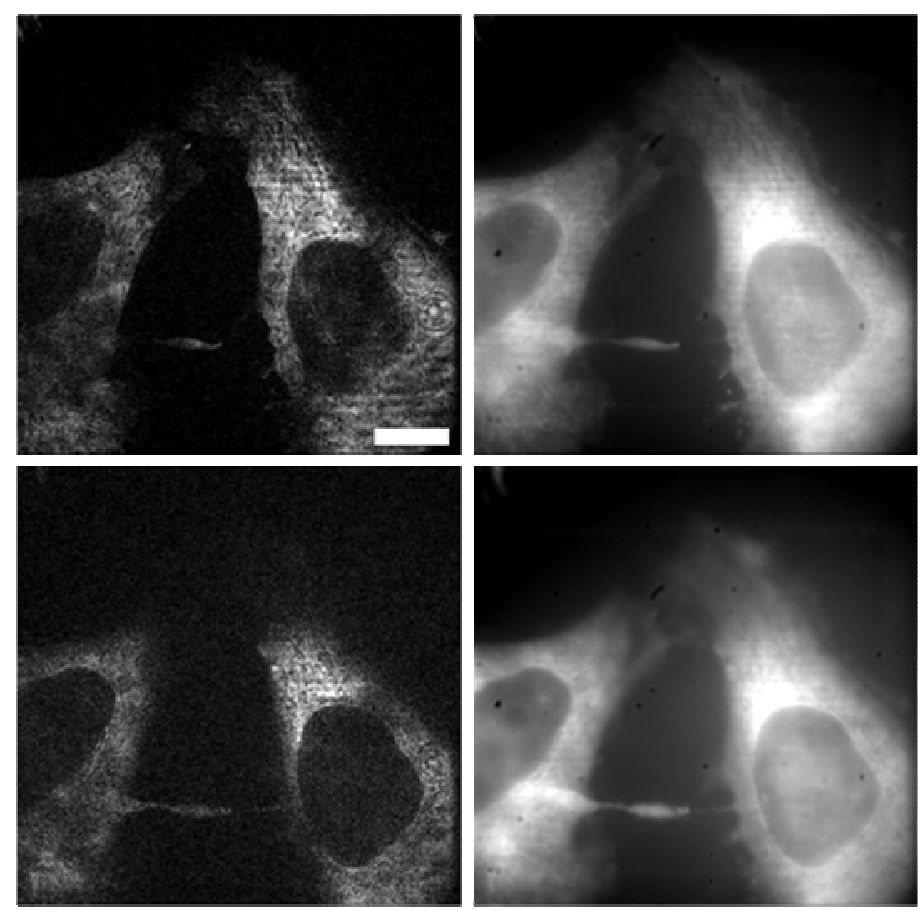